\title{
RESEARCH
}

Open Access

\section{Epidemiological factors of septoria tritici blotch (Zymoseptoria tritici) in durum wheat (Triticum turgidum) in the highlands of Wollo, Ethiopia}

Bogale Nigir Hailemariam $^{1 *}$ (D) Yosef Geberehawariat Kidane ${ }^{2}$ and Amare Ayalew ${ }^{3}$

\begin{abstract}
Background: Septoria tritici blotch (STB) (Zymoseptoria tritici) is a major disease of durum wheat, an economic crop grown in the highlands of Wollo in Ethiopia.

Methods: To determine the status of this disease, we conducted surveys in five districts of Wollo (Meket, Woreilu, Wadila, Jama, and Dessie Zuria) during the 2015 cropping season. We visited 75 farm plots to determine the prevalence, incidence, and severity of STB.

Results: STB prevalence varied among locations, genotypes, planting dates, growth stages, previous crops, plant population, weed population, and soil types. Similarly, disease intensity also varied along all independent variables. The level of incidence was high in all the visited districts, and the level severity ranged from 9.9 to $59.3 \%$ while the incidence varied from 50 to 100\%. The mean differences in incidence and severity within the districts' variable classes, altitude, varieties, growth stage, plant population, planting date, previous crop, weed population, and soil type were high. The independent variables, districts, altitude, varieties, growth stage, plant population, planting date, previous crops, weed population, and soil type, were significantly associated with high incidence and severity of STB as single predictor in the logistic regression model. A reduced multiple variable model was fitted using districts, altitude, varieties, growth stage, plant population, planting date, previous crop, weed population, and soil type as independent variables. High incidence $(>50 \%)$ and severity $(>25 \%)$ had a high probability of association to all independent variables, except previous crop. Low disease incidence $(\leq 50 \%)$ and low disease severity $(\leq 25 \%)$ had high probability of association to the previous crop.
\end{abstract}

Conclusion: Environmental variables, cultivation practice, and responses were important for the development of STB. Therefore, these factors must be considered in designing strategies for the effective management of STB.

Keywords: Durum wheat, Zymoseptoria tritici, Septoria tritici blotch

\footnotetext{
* Correspondence: bogale84@gmail.com

${ }^{1}$ Sirinka Agricultural Research Center, Woldia, Ethiopia

Full list of author information is available at the end of the article
}

\section{Springer Open}

( ) The Author(s). 2020 Open Access This article is licensed under a Creative Commons Attribution 4.0 International License, which permits use, sharing, adaptation, distribution and reproduction in any medium or format, as long as you give appropriate credit to the original author(s) and the source, provide a link to the Creative Commons licence, and indicate if changes were made. The images or other third party material in this article are included in the article's Creative Commons licence, unless indicated otherwise in a credit line to the material. If material is not included in the article's Creative Commons licence and your intended use is not permitted by statutory regulation or exceeds the permitted use, you will need to obtain permission directly from the copyright holder. To view a copy of this licence, visit http://creativecommons.org/licenses/by/4.0/. 


\section{Introduction}

Zymoseptoria tritici is the causal agent for septoria tritici blotch (STB) that attacks durum wheat (Triticum turgidum L. subsp. durum) (Eyal et al. 1985). It is widely distributed in the Mediterranean basin, East Africa, South America, Australia, and Western Europe (Saari and Wilcoxson 1974; Rajaram and Dubin 1977; Polley and Thomas 1991). In the USA, septoria tritici blotch is second only to wheat stem rust in terms of importance, and it is the number one disease of wheat in Russia and many countries of Western Europe (Ponomarenko et al. 2011). This fungus is essentially a leaf pathogen and rarely causes blotches on glumes. In the initial stages of the disease, the blotches are clear yellow, small, globular, or oblong, sometimes narrower than the spots. Severe epidemics of septoria tritici blotch can reduce wheat yields by 35 to 50\% worldwide (Ponomarenko et al. 2011).

Studies focusing on septoria tritici blotch of durum wheat have not been conducted in the highlands of Wollo, where durum wheat is grown in large scale. Further, cultural practices, altitude, and soil types, which affect septoria tritici blotch development and response of genotypes to the disease, are seldom reported in the region. Information on occurrence, distribution, and factors affecting the disease is important to develop management options.

The improvement of durum wheat based on improved varieties of local collections is the main component for the management of septoria tritici blotch (Blixt 1988; Damania et al. 1992). These genetic resources contain several important agronomic and resistance genes (Grausgrubera et al. 2005; Yahyaoui et al. 2006). The availability of such germplasm depends on the identification of the sources of diversification (Devra and Hodgkin 1999; Ganeva et al. 2011), especially within the primary and secondary diversity centers which are characterized by large diversity. Ethiopia is one of the centers of diversity for durum wheat (CSA (Central Statistical Agency) 2009). The local germplasm is adapted to a wide range of environments and carries a large reservoir of useful genes (Zelalem 1989; Cherdouh et al. 2005). Resistance to septoria tritici blotch (Zymoseptoria tritici) is generally expressed through the reduction of the foliar area covered with pycnidia and/or necrosis.

Given the economic relevance, the development of strategies to manage septoria tritici blotch is of great importance. Generating strategies and information on occurrence, distribution, and factors affecting the disease

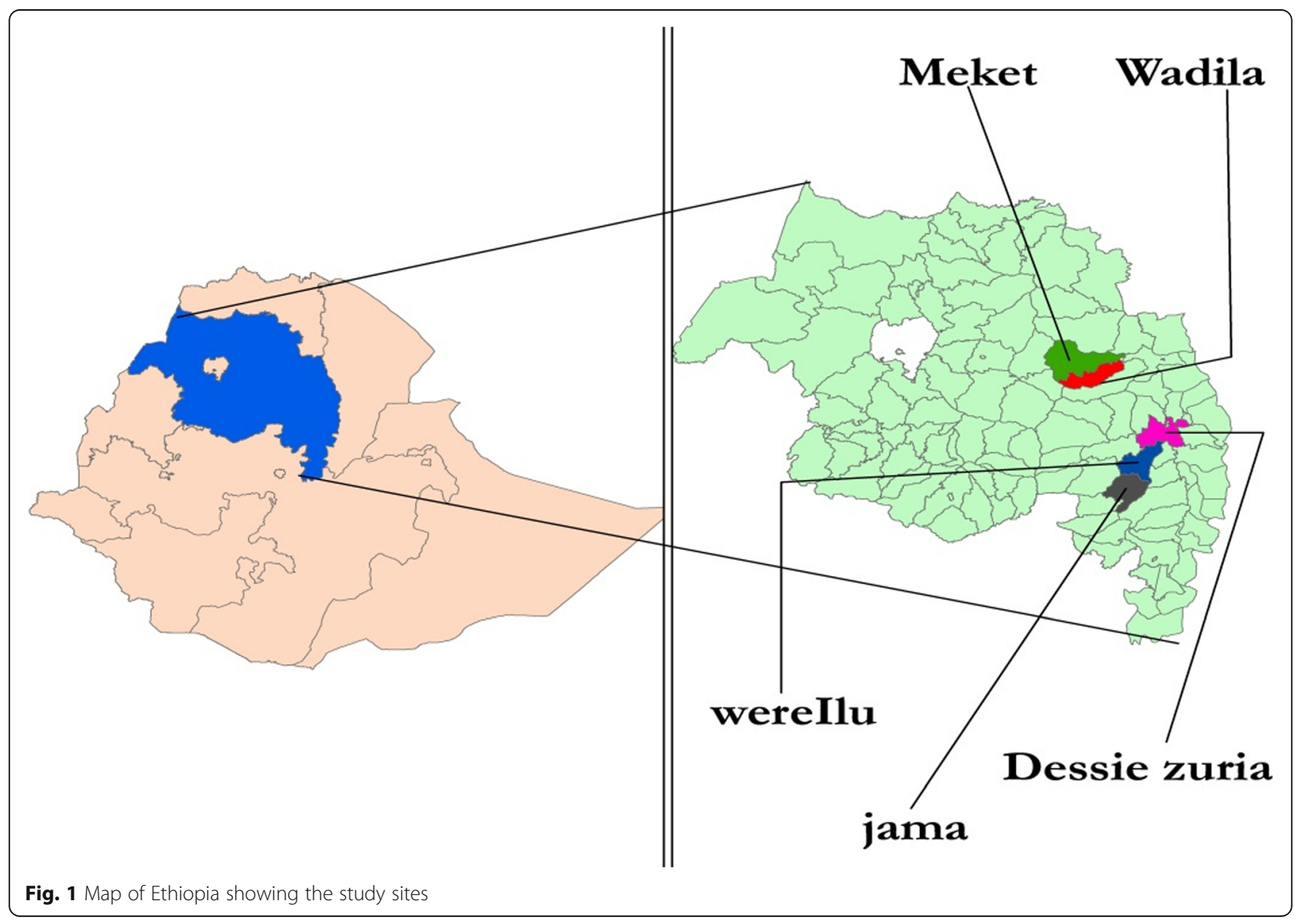


are important to develop disease management options. Among the strategies to combat diseases, genetic resistance stands out as the most appropriate disease management option in terms of stability, economy, and environmental impact.

The high level of diversity in Triticum turgidum subsp. durum landraces allows breeders and pathologists to make selection for different traits of economic importance. However, information about the septoria tritici blotch and the reaction of genotypes to the disease in the highlands of Wollo is not available. Therefore, the objective of this study was to determine the incidence and severity of septoria tritici blotch on durum wheat in the highlands of Wollo.

\section{Materials and methods}

\section{Survey area and sampling procedures}

Field surveys were conducted in five districts of Wollo highlands, namely Jama, Dessie Zuria, Wadila, Woreilu, and Meket (Fig. 1). The surveys were conducted in the area, from October to November 2015, which covers the main cropping season. From each district, 15 representative farm plots were assessed and plant samples were taken randomly at fixed interval of 5 up to $10 \mathrm{~km}$. A total of 75 fields (15 per district) were assessed.

\section{Crop and disease assessments}

The prevalence of septoria tritici blotch was computed based on the presence and absence of disease within the farm plot. Disease incidence and severity were assessed visually by walking in each field in an " $\mathrm{X}$ " fashion and taking 10 random plants within a quadrat $(0.5 \mathrm{~m} \times 0.5$ $\mathrm{m})$. Disease incidence was calculated as the percentage of the number of infected plants by the total number of plants assessed. Disease severity was scored based on the double-digit recording system (00 to 99), modified version of Saari and Prescott's scale (Saari and Prescott 1975; Eyal et al. 1987; Sharma and Duveiller 2007) for wheat foliar diseases. The first digit $(D 1)$ indicates disease progress in plant height, and the second digit $(D 2)$ refers to severity measured as the diseased leaf area.

In addition to disease data, other supplementary information was collected, including soil type, durum wheat variety name, planting date, altitude, plant population, weed population, previous crop, and growth stage. The plant and weed population was counted in each quadrat. The mean plant and weed population density was obtained by averaging five quadrats, and the weed density was recorded as low (20-40 weeds), medium (41-60 weeds), and high (61-110 weeds $\mathrm{m}^{-2}$ ) and plant population as high $\left(>210\right.$ plants $\left.\mathrm{m}^{-2}\right)$, low $\left(<140\right.$ plants $\left.\mathrm{m}^{-2}\right)$, and medium (141-210 plants $\mathrm{m}^{-2}$ ) (Woldeab et al. 2007). Information on variety type, previous crop, and planting date was obtained from growers through interview. The growth stage of the crop was recorded using
Zadoks' scale. It consists of a two-digit code: the first digit referring to the principal stage of development (from 0 to 9 , germination to kernel ripening) and the second digit allowing subdivision of the bespoke principal (Zadoks and Schein 1979; Eyal et al. 1987).

\section{Disease and crop parameters}

Disease severity was assessed on five randomly taken plants in the two rows of every plot, and data were recorded and assessed at late milk stage of the crop (Eyal et al. 1987).

The disease severity was scored visually using a double-digit (00 to 99) recording system. The first digit $(D 1)$ indicates disease progress in plant height, and the second digit $(D 2)$ refers to severity measured as the diseased leaf area.

\section{Statistical analysis}

For each score, percentage of disease severity was estimated based on the following formula (Sharma and Duveiller 2007):

$$
\text { Severity }=(D 1 / 9) \times(D 2 / 9) \times 100
$$

where $D 1=$ the disease progress in plant height and $D 2$ $=$ the severity measured as the diseased leaf area.

Simple descriptive statistics were used to summarize data obtained from field surveys by using proc mean SAS procedure (SAS Institute 2003). The values corresponding to each independent variable represented the frequency of fields falling in the different boundaries and were used to analyze the association of septoria tritici blotch incidence and severity with the independent variables and variable class using stepwise logistic regression of SAS procedure (SAS Institute 1993; 2003). The procedure has been used by many authors to evaluate the association of disease incidence and severity of the different independent variables (Fininsa and Yuen 2001a; Woldeab et al. 2007; Zewde et al. 2007). The logit link function was used in this binomially distributed data to determine the association of the independent variable to the response variable (Fininsa and Yuen 2001b; Zewde et al. 2007). The binary outcome was the probability that durum wheat septoria tritici blotch incidence exceeds $50 \%$ and severity exceeds $25 \%$ in a given durum wheat field (Table 1). The likelihood ratio statistic was used to examine the importance of variables and was tested against a chi-square (McCullagh and Nelder 1989).

\section{Results}

Prevalence of septoria tritici blotch

We found STB in all our study sites (Table 2), but the highest prevalence was recorded at Wadila $(87 \%$ of the fields showing the disease), followed by Jama (80\%), 
Table 1 Independent variables by disease contingency for logistic regression analysis of durum wheat septoria tritici blotch in the highlands of Wollo

\begin{tabular}{|c|c|c|c|c|c|c|c|c|c|c|c|}
\hline \multirow[t]{2}{*}{ Variable } & \multirow{2}{*}{$\begin{array}{l}\text { Variable } \\
\text { class }\end{array}$} & \multicolumn{2}{|c|}{ Incidence } & \multicolumn{2}{|c|}{ Severity } & \multirow[t]{2}{*}{ Variable } & \multirow[t]{2}{*}{ Variable class } & \multicolumn{2}{|c|}{ Incidence } & \multicolumn{2}{|c|}{ Severity } \\
\hline & & $\leq 50$ & $>50$ & $\leq 25$ & $>25$ & & & $\leq 50$ & $>50$ & $\leq \mathbf{2 5}$ & $>25$ \\
\hline \multirow[t]{5}{*}{ District } & Dessie Zuria & 15 & 0 & 15 & 0 & Plant population & High & 12 & 13 & 16 & 9 \\
\hline & Jama & 13 & 2 & 15 & 0 & & Low & 14 & 1 & 15 & 0 \\
\hline & Meket & 7 & 8 & 8 & 7 & & Medium & 28 & 7 & 31 & 4 \\
\hline & Wadila & 9 & 6 & 11 & 4 & Planting date & August & 13 & 2 & 14 & 1 \\
\hline & Woreilu & 10 & 5 & 13 & 2 & & Early July & 13 & 6 & 14 & 5 \\
\hline \multirow[t]{2}{*}{ Altitude } & $\leq 2700$ masl & 32 & 9 & 38 & 3 & & June & 5 & 7 & 6 & 6 \\
\hline & > 2700 masl & 22 & 12 & 24 & 10 & & Late July & 12 & 3 & 15 & 0 \\
\hline \multirow[t]{11}{*}{ Variety } & Abesha & 3 & 3 & 6 & 0 & & Mid-July & 11 & 3 & 13 & 1 \\
\hline & Enat & 6 & 0 & 6 & 0 & Previous crop & Barley & 8 & 0 & 8 & 0 \\
\hline & Enzosh & 5 & 2 & 6 & 1 & & Legume & 18 & 2 & 19 & 1 \\
\hline & Firno & 1 & 6 & 2 & 5 & & Tef & 7 & 2 & 8 & 1 \\
\hline & Gojam & 3 & 0 & 3 & 0 & & Wheat & 21 & 17 & 27 & 11 \\
\hline & Gundile & 5 & 1 & 5 & 1 & Weed population & High (> 61 weeds $\mathrm{m}^{2}$ ) & 2 & 9 & 4 & 7 \\
\hline & Key sindie & 9 & 4 & 9 & 4 & & Low $\left(<40\right.$ weeds $\left.\mathrm{m}^{2}\right)$ & 39 & 5 & 41 & 3 \\
\hline & Laste & 6 & 3 & 7 & 2 & & Medium (41-60 weeds $\mathrm{m}^{2}$ ) & 17 & 7 & 17 & 3 \\
\hline & Nesh & 3 & 0 & 3 & 0 & Soil type & Red & 8 & 10 & 11 & 7 \\
\hline & Nich jiru & 9 & 1 & 10 & 0 & & Gray & 12 & 7 & 14 & 5 \\
\hline & Tikur jiru & 4 & 1 & 5 & 0 & & Black & 34 & 4 & 37 & 1 \\
\hline \multirow[t]{4}{*}{ Growth stage } & Early flower & 14 & 3 & 16 & 1 & & & & & & \\
\hline & Flower & 1 & 0 & 1 & 0 & & & & & & \\
\hline & Late milk & 8 & 0 & 8 & 0 & & & & & & \\
\hline & Milk & 31 & 18 & 37 & 12 & & & & & & \\
\hline
\end{tabular}

Woreilu (80\%), and Meket (80\%) while the lowest (40\%) was recorded at Dessie Zuria. Septoria tritici blotch prevalence varied among genotypes, planting dates, growth stages, previous crops, plant populations, weed populations, and soil types. The highest STB prevalence (100\%) was recorded on Abesha sindie and Firno local varieties, while the lowest STB prevalence (33\%) was registered in Enat, Gojam gura, and Nech sindie varieties.

Previous crops were important in determining the prevalence of STB. Highest STB prevalence (92\%) was recorded on previously grown wheat field, while the lowest prevalence (50\%) was recorded on previously legume and barley grown fields. Varied levels of STB prevalence were registered in different planting times, where highest STB prevalence (92\%) was recorded in June, while lowest prevalence (53\%) in August planting time.

Highest $(100 \%)$ STB prevalence was recorded on the high plant population compared to a low plant population (47\%). Similarly, highest (100\%) weed population resulted in high STB prevalence compared to low weed population (59\%). Varied levels of STB prevalence were also recorded among soil types that red soil type showed the highest STB prevalence (94\%), while black soil showed the lowest prevalence $(60 \%)$.

\section{Intensity of septoria tritici blotch}

Mean incidence and severity of septoria tritici blotch on durum wheat for different independent variables were presented (Table 2). The survey results showed that the highest (55.3\%) STB mean incidence was recorded at Meket, followed by Wadila (42.7\%), while the lowest (16\%) was recorded at Dessie Zuria. Similarly, the maximum mean STB severity $(21.7 \%)$ was recorded at Meket, followed by Wadila (14.2\%), while Dessie Zuria showed the least STB severity (2.5\%).

The disease intensity varied along altitudinal range. When altitude increased, the level of mean STB incidence as well as severity increased. Generally, it was more severe in districts with altitude $>2700$ masl than lower elevations and the maximum severity level (59\%) was recorded in areas with altitude $>2700$ masl (Table 2). Similarly, there was an increased incidence level with increase in altitude. The maximum incidence was recorded in areas with $>2700$ masl (Table 2). 
Table 2 Prevalence, mean incidence, and severity of septoria tritici blotch on durum wheat under different independent variables

\begin{tabular}{|c|c|c|c|c|c|c|c|c|c|c|}
\hline \multirow[t]{3}{*}{ Variable } & \multirow[t]{3}{*}{ Variable class } & \multirow{3}{*}{$\begin{array}{l}\text { Prevalence } \\
\text { (\%) }\end{array}$} & \multicolumn{8}{|c|}{ Septoria tritici blotch } \\
\hline & & & \multicolumn{4}{|c|}{ Incidence } & \multicolumn{4}{|c|}{ Severity } \\
\hline & & & Mean & SD & Min & Max & Mean & SD & Min & Max \\
\hline \multirow[t]{5}{*}{ District } & Dessie Zuria & 40 & 16.7 & 21.9 & 0 & 50 & 2.5 & 3.6 & 0 & 9.9 \\
\hline & Jama & 80 & 34.0 & 22.0 & 0 & 70 & 6.2 & 5.2 & 0 & 14.8 \\
\hline & Meket & 80 & 55.3 & 40.7 & 0 & 100 & 21.7 & 22.1 & 0 & 59.3 \\
\hline & Wadila & 87 & 42.7 & 27.1 & 0 & 90 & 14.2 & 12.7 & 0 & 39.5 \\
\hline & Woreilu & 80 & 38.0 & 26.2 & 0 & 80 & 12.3 & 13.2 & 0 & 49.4 \\
\hline \multirow[t]{2}{*}{ Altitude } & $\leq 2700$ masl & 75.6 & 35.36 & 25.40 & 0 & 80 & 8.35 & 9.72 & 0 & 49 \\
\hline & > 2700 masl & 70.6 & 39.70 & 35.80 & 0 & 100 & 15.0 & 18.0 & 0 & 59 \\
\hline \multirow[t]{11}{*}{ Variety } & Abesha & 100 & 50.0 & 12.6 & 30 & 60 & 11.0 & 4.7 & 3.7 & 17.3 \\
\hline & Enat & 33 & 10.0 & 15.5 & 0 & 30 & 0.8 & 1.3 & 0 & 2.5 \\
\hline & Enzosh & 71 & 32.9 & 29.3 & 0 & 70 & 9.9 & 10.8 & 0 & 29.6 \\
\hline & Firno & 100 & 74.3 & 17.2 & 50 & 100 & 28.4 & 13.0 & 9.9 & 49.4 \\
\hline & Gojam gura & 33 & 10.0 & 17.3 & 0 & 30 & 0.8 & 1.4 & 0 & 2.5 \\
\hline & Gundile & 67 & 31.7 & 27.1 & 0 & 70 & 11.1 & 11.9 & 0 & 29.6 \\
\hline & Key sindie & 77 & 41.5 & 37.8 & 0 & 100 & 16.2 & 20.8 & 0 & 59.3 \\
\hline & Laste & 89 & 51.1 & 28.5 & 0 & 100 & 16.7 & 18.8 & 0 & 49.4 \\
\hline & Nech & 33 & 6.7 & 11.5 & 0 & 20 & 0.8 & 1.4 & 0 & 2.5 \\
\hline & Nech jiru & 70 & 28.0 & 24.4 & 0 & 60 & 5.6 & 5.8 & 0 & 14.8 \\
\hline & Tikur jiru & 80 & 34.0 & 26.1 & 0 & 70 & 5.2 & 4.5 & 0 & 12.3 \\
\hline \multirow[t]{5}{*}{ Planting date } & August & 53 & 24.0 & 25.9 & 0 & 70 & 6.2 & 8.8 & 0 & 29.6 \\
\hline & Early July & 84 & 43.2 & 28.7 & 0 & 100 & 13.7 & 14.5 & 0 & 49.4 \\
\hline & June & 92 & 66.7 & 32.0 & 0 & 100 & 27.1 & 21.0 & 0 & 59.3 \\
\hline & Late July & 67 & 28.7 & 23.9 & 0 & 60 & 4.6 & 5.3 & 0 & 17.3 \\
\hline & Mid-July & 71 & 27.9 & 26.4 & 0 & 70 & 7.6 & 8.6 & 0 & 29.6 \\
\hline \multirow[t]{4}{*}{ Previous crop } & Barley & 50 & 17.5 & 20.5 & 0 & 50 & 2.6 & 37 & 0 & 9.9 \\
\hline & Legume & 50 & 17.5 & 23.4 & 0 & 80 & 4.0 & 6.5 & 0 & 25.9 \\
\hline & Tef & 67 & 30.0 & 28.7 & 0 & 70 & 7.4 & 9.1 & 0 & 25.9 \\
\hline & Wheat & 92 & 53.7 & 27.0 & 0 & 100 & 18.0 & 16.7 & 0 & 59.3 \\
\hline \multirow[t]{4}{*}{ Growth stage } & Early flower & 65 & 27.1 & 24.9 & 0 & 70 & 6.1 & 8.1 & 0 & 29.6 \\
\hline & Flower & 100 & 30.0 & & 30 & 30 & 7.4 & & 7.4 & 7.40 \\
\hline & Late milk & 37 & 17.5 & 24.3 & 0 & 50 & 3.1 & 4.3 & 0 & 9.9 \\
\hline & Milk & 82 & 44.3 & 31.3 & 0 & 100 & 14.6 & 16.2 & 0 & 59.3 \\
\hline \multirow[t]{3}{*}{ Plant population } & High (> 210 plants $\mathrm{m}^{-2}$ ) & 100 & 60.0 & 23.2 & 20 & 100 & 18.9 & 17.2 & 2.5 & 59.3 \\
\hline & Low $\left(<140\right.$ plants $\left.\mathrm{m}^{-2}\right)$ & 47 & 26.5 & 29.2 & 0 & 70 & 6.0 & 8.8 & 0 & 25.9 \\
\hline & Medium $\left(141-210 \mathrm{~m}^{-2}\right)$ & 66 & 32.3 & 32.2 & 0 & 100 & 10.7 & 16.0 & 0 & 59.3 \\
\hline \multirow[t]{3}{*}{ Weed population } & High (> 61 weeds $\mathrm{m}^{-2}$ ) & 100 & 73.6 & 22.9 & 30 & 100 & 29.7 & 17.6 & 7.4 & 59.3 \\
\hline & Low $\left(<40\right.$ weeds $\left.\mathrm{m}^{-2}\right)$ & 59 & 23.4 & 23.6 & 0 & 70 & 5.4 & 7.8 & 0 & 29.6 \\
\hline & Medium (41-60 weeds $\mathrm{m}^{-2}$ ) & 90 & 48.0 & 27.1 & 0 & 100 & 14.5 & 14.9 & 0 & 49.4 \\
\hline \multirow[t]{3}{*}{ Soil type } & Red & 94 & 58.3 & 30.0 & 0 & 100 & 18.9 & 15.7 & 0 & 49.4 \\
\hline & Gray & 79 & 44.2 & 31.5 & 0 & 100 & 17.3 & 18.8 & 0 & 59.3 \\
\hline & Black & 60 & 23.9 & 23.1 & 0 & 70 & 4.8 & 6.4 & 0 & 29.6 \\
\hline
\end{tabular}


In this field survey, varied levels of mean STB incidence and severity reaction were registered among durum wheat varieties. Firno local variety scored the highest $(74.3 \%)$ mean disease incidence, while Nech sindie scored the lowest (6.7\%) mean disease incidence. Nech sindie, Enat sindie, and Gojam gura varieties showed low disease incidence, and the highest disease severity was observed in Firno variety.

Cultural practices also significantly affected the incidence and severity of STB disease on durum wheat. The highest mean disease incidence (60.0\%) and severity (18.9\%) of STB were recorded in higher plant population, whereas lower plant population showed $26.5 \%$ incidence and 6\% severity. Similarly, the highest mean disease incidence (73.6\%) and severity (29.7\%) of STB were recorded in high weed density compared with low weed density (23.4\% incidence and $5.4 \%$ disease severity). Previous crops, viz. wheat, tef, barley, and legumes, showed variation in STB. The highest disease incidence $(53.7 \%)$ and severity (18\%) were observed in previously wheat grown fields.

In this study, the planting time affected the development STB on durum wheat. Higher disease incidence $(66.7 \%)$ and severity (18\%) were observed in early durum wheat planted fields than late planted fields.

Durum wheat fields sown on red soil type showed the highest STB mean incidence (53.3\%) and severity (18.9\%) while black soil type showed the lowest disease incidence (23.9\%) and severity (4.8\%).

\section{Association of durum wheat septoria tritici blotch intensity with environmental factors and cultural practices}

The probability of septoria tritici blotch (STB) associated with the independent variables was presented (Table 3). Durum wheat STB mean incidence and severity were significantly associated with all independent variables: district, altitude, variety, growth stage, plant population, planting date, previous crop, weed density, and soil type as single predictors in the logistic regression (Table 3). Analysis of deviance, natural logarithms of odds ratio, and standard error of added variables in a reduced model analyzing durum wheat STB incidence and severity were also presented (Tables 4 and 5). All independent variables were tested in a reduced multiple model.

\section{Association of septoria tritici blotch with districts and altitude}

This septoria tritici blotch (STB) survey result indicated that the disease was widely distributed in all districts and showed variation. The probability of association of district and altitude range to high STB incidence $(>50 \%)$ and high severity $(>25 \%)$ was significant with low $(\leq 0 \%)$ disease incidence and low $(\leq 25 \%)$ severity (Table 3$)$. Meket district and lower altitude ( $\leq 2700$ masl) had a high probability of association to high STB incidence and severity. The probability of occurrence of $>50 \%$ incidence was 1.8 times higher in $\leq 2700$ masl altitude than in $>2700$ masl altitude (Table 4). However, $>25 \%$ severity was more associated with Woreilu and Jama district than Meket and $\leq 25 \%$ severity positively associated with $\leq 2700$ masl than $>2700$ masl altitude (Table 5).

\section{Association of septoria tritici blotch with varieties and growth stage}

Different durum wheat varieties were analyzed by logistic regression model as a single predictor, and the result showed that there was a highly significant association with more than $50 \%$ incidence and above $25 \%$ severity (Table 3). In the reduced model, Abesha, Enat, Enzosh, Firno, and Lastie wheat varieties were associated with the probability of high incidence occurrence, but Gojam gura, Gundile, Key sindie, Nech sindie, and Nech jiru

Table 3 Independent variables used in logistic regression modeling durum wheat incidence and severity and likelihood ratio test (LRT) for nine variables as single predictors of disease outcome

\begin{tabular}{|c|c|c|c|c|c|}
\hline \multirow{2}{*}{$\begin{array}{l}\text { Independent } \\
\text { variable }\end{array}$} & \multirow[t]{2}{*}{ df } & \multicolumn{2}{|c|}{ Septoria tritici blotch incidence LRT $>50 \%$} & \multicolumn{2}{|c|}{ Septoria tritici blotch severity LRT $>25 \%$} \\
\hline & & DR & $\operatorname{Pr}>x^{2}$ & DR & $\operatorname{Pr}>x^{2}$ \\
\hline District & 4 & 534.21 & $<.0001$ & 356.02 & $<.0001$ \\
\hline Altitude & 1 & 128.55 & $<.0001$ & 6.10 & 0.0135 \\
\hline Variety & 10 & 931.59 & $<.0001$ & 372.15 & $<.0001$ \\
\hline Growth stage & 3 & 53.77 & $<.0001$ & 16.18 & 0.001 \\
\hline Plant population & 2 & 474.36 & $<.0001$ & 165.41 & $<.0001$ \\
\hline Planting date & 4 & 33.04 & $<.0001$ & 15.49 & 0.0038 \\
\hline Previous crop & 3 & 222.05 & $<.0001$ & 181.28 & $<.0001$ \\
\hline Weed population & 2 & 13.88 & 0.001 & 32.06 & $<.0001$ \\
\hline Soil type & 2 & 59.69 & $<.0001$ & 11.45 & 0.0033 \\
\hline
\end{tabular}

$d f$ degrees of freedom, $D R$ deviance reduction, $\operatorname{Pr}$ probability of a $X^{2}$ value exceeding the deviance reduction 
Table 4 Analysis of deviance, natural logarithms of odds ratio, and standard error of added variables in a reduced model analyzing septoria tritici blotch incidence

\begin{tabular}{|c|c|c|c|c|c|c|c|c|}
\hline Added variable & Residual deviance & df & Deviance reduction & $\operatorname{Pr}>x^{2}$ & Variable class & Estimate loge & SE & Odds ratio \\
\hline Intercept & 3752.19 & & & & & -0.73 & 0.37 & 0.48 \\
\hline \multirow[t]{5}{*}{ District } & 3217.99 & 4 & 534.21 & $<.0001$ & Jama & -0.74 & 0.34 & 0.48 \\
\hline & & & & & Wadila & -0.35 & 0.15 & 0.70 \\
\hline & & & & & Woreilu & -0.39 & 0.31 & 0.68 \\
\hline & & & & & Dessie Zuria & -1.92 & 0.31 & 0.15 \\
\hline & & & & & Meket & 0.00 & 0.00 & 1 \\
\hline \multirow[t]{2}{*}{ Altitude } & 3089.44 & 1 & 128.55 & $<.0001$ & $\leq 2700$ masl & 0.61 & 0.12 & 1.85 \\
\hline & & & & & $>2700$ masl & 0.00 & 0.00 & 1 \\
\hline \multirow[t]{11}{*}{ Variety } & 2157.84 & 10 & 931.59 & $<.0001$ & Abesha & 0.36 & 0.28 & 1.43 \\
\hline & & & & & Enat & 0.34 & 0.33 & 1.41 \\
\hline & & & & & Enzosh & 0.10 & 0.35 & 1.10 \\
\hline & & & & & Firno & 0.42 & 0.37 & 1.52 \\
\hline & & & & & Gojam & -0.10 & 0.36 & 0.91 \\
\hline & & & & & Gundile & -0.07 & 0.23 & 0.93 \\
\hline & & & & & Key & -0.21 & 0.33 & 0.81 \\
\hline & & & & & Laste & 0.38 & 0.24 & 1.47 \\
\hline & & & & & Nech & -1.12 & 0.46 & 0.33 \\
\hline & & & & & Nech jiru & -0.32 & 0.13 & 0.73 \\
\hline & & & & & Tikur jiru & 0.00 & 0.00 & 1 \\
\hline \multirow[t]{4}{*}{ Growth stage } & 2104.07 & 3 & 53.77 & $<.0001$ & Early flowering & -0.35 & 0.12 & 0.70 \\
\hline & & & & & Flower & -0.20 & 0.31 & 0.82 \\
\hline & & & & & Late milk & 0.15 & 0.19 & 1.16 \\
\hline & & & & & Milk & 0.00 & 0.00 & 1 \\
\hline \multirow[t]{3}{*}{ Plant population } & 1629.71 & 2 & 474.36 & $<.0001$ & High (> 210 plants $\mathrm{m}^{-2}$ ) & 0.44 & 0.09 & 1.56 \\
\hline & & & & & Low $\left(<140\right.$ plants $\left.\mathrm{m}^{-2}\right)$ & -0.93 & 0.12 & 0.39 \\
\hline & & & & & Medium (141-210 $\left.\mathrm{m}^{-2}\right)$ & 0.00 & 0.00 & 1 \\
\hline \multirow[t]{5}{*}{ Planting date } & 1596.67 & 4 & 33.04 & $<.0001$ & August & 0.41 & 0.13 & 1.50 \\
\hline & & & & & Early July & 0.02 & 0.12 & 1.02 \\
\hline & & & & & June & 0.77 & 0.15 & 2.16 \\
\hline & & & & & Late July & 0.13 & 0.14 & 1.14 \\
\hline & & & & & Mid-July & 0.00 & 0.00 & 1 \\
\hline \multirow[t]{4}{*}{ Previous crop } & 1374.62 & 2 & 222.05 & $<.0001$ & Barley & -1.75 & 0.18 & 0.17 \\
\hline & & & & & Legume & -1.18 & 0.12 & 0.31 \\
\hline & & & & & Tef & -0.59 & 0.12 & 0.56 \\
\hline & & & & & Wheat & 0.00 & 0.00 & 1 \\
\hline \multirow[t]{3}{*}{ Weed population } & 1360.73 & 2 & 13.88 & 0.001 & High (> 61 weeds $\mathrm{m}^{-2}$ ) & 0.58 & 0.14 & 1.79 \\
\hline & & & & & Low $\left(<40\right.$ weeds $\left.\mathrm{m}^{-2}\right)$ & 0.42 & 0.11 & 1.53 \\
\hline & & & & & Medium (41-60 weeds $\mathrm{m}^{-2}$ ) & 0.00 & 0.00 & 1 \\
\hline \multirow[t]{3}{*}{ Soil type } & 1301.05 & 2 & 59.69 & $<.0001$ & Red & 0.94 & 0.15 & 2.55 \\
\hline & & & & & Gray & 0.79 & 0.11 & 2.21 \\
\hline & & & & & Black & 0.00 & 0.00 & 1 \\
\hline
\end{tabular}


Table 5 Analysis of deviance, natural logarithms of odds ratio, and standard error of added variables in a reduced model analyzing septoria tritici blotch severity

\begin{tabular}{|c|c|c|c|c|c|c|c|c|}
\hline Added variable & $\begin{array}{l}\text { Residual } \\
\text { deviance }\end{array}$ & df & $\begin{array}{l}\text { Deviance } \\
\text { reduction }\end{array}$ & $\operatorname{Pr}>x^{2}$ & Variable class & $\begin{array}{l}\text { Estimate loge (odds } \\
\text { ratio) }\end{array}$ & SE & $\begin{array}{l}\text { Odds } \\
\text { ratio }\end{array}$ \\
\hline Intercept & 1378.9 & & & & & -3.42 & 0.54 & 0.03 \\
\hline \multirow[t]{5}{*}{ District } & \multirow[t]{5}{*}{1022.9} & \multirow[t]{5}{*}{4} & \multirow[t]{5}{*}{356.02} & \multirow{5}{*}{$\begin{array}{l}< \\
0.0001\end{array}$} & Jama & 0.45 & 0.50 & 1.56 \\
\hline & & & & & Wadila & 0.69 & 0.19 & 0.50 \\
\hline & & & & & Woreilu & 1.19 & 0.38 & 3.30 \\
\hline & & & & & Dessie Zuria & -1.64 & 0.50 & 0.19 \\
\hline & & & & & Meket & 0.00 & 0.00 & 1 \\
\hline \multirow[t]{2}{*}{ Altitude } & \multirow[t]{2}{*}{1016.81} & \multirow[t]{2}{*}{1} & \multirow[t]{2}{*}{6.10} & \multirow[t]{2}{*}{0.0135} & $\leq 2700$ masl & -0.30 & 0.16 & 0.74 \\
\hline & & & & & $>2700$ masl & 0.00 & 0.00 & 1 \\
\hline \multirow[t]{11}{*}{ Variety } & \multirow[t]{11}{*}{644.66} & \multirow[t]{11}{*}{10} & \multirow[t]{11}{*}{372.15} & \multirow[t]{11}{*}{$<.0001$} & Abesha & 0.85 & 0.50 & 2.33 \\
\hline & & & & & Enat & 0.55 & 0.70 & 1.73 \\
\hline & & & & & Enzosh & 2.58 & 0.50 & 13.14 \\
\hline & & & & & Firno & 2.57 & 0.56 & 13.10 \\
\hline & & & & & Gojam & -1.27 & 0.83 & 0.28 \\
\hline & & & & & Gundile & 0.40 & 0.45 & 1.50 \\
\hline & & & & & Key & 1.32 & 0.50 & 3.75 \\
\hline & & & & & Laste & 0.98 & 0.41 & 2.67 \\
\hline & & & & & Nech & 1.37 & 0.88 & 3.95 \\
\hline & & & & & Nech jiru & 0.00 & 0.27 & 1.00 \\
\hline & & & & & Tikur jiru & 0.00 & 0.00 & 1 \\
\hline \multirow[t]{4}{*}{ Growth stage } & \multirow[t]{4}{*}{628.48} & \multirow[t]{4}{*}{3} & \multirow[t]{4}{*}{16.18} & \multirow[t]{4}{*}{0.001} & Early flowering & -0.30 & 0.21 & 0.74 \\
\hline & & & & & Flower & 0.69 & 0.51 & 1.99 \\
\hline & & & & & Late milk & 0.86 & 0.42 & 2.37 \\
\hline & & & & & Milk & 0.00 & 0.00 & 1.00 \\
\hline \multirow[t]{3}{*}{ Plant population } & \multirow[t]{3}{*}{463.07} & \multirow[t]{3}{*}{2} & \multirow[t]{3}{*}{165.41} & $<.0001$ & High (> 210 plants $\mathrm{m}^{2}$ ) & 0.32 & 0.16 & 1.38 \\
\hline & & & & & Low $\left(<140\right.$ plants $\left.\mathrm{m}^{2}\right)$ & -0.80 & 0.23 & 0.45 \\
\hline & & & & & Medium (141-210) & 0.00 & 0.00 & 1.00 \\
\hline Planting date & 447.58 & 4 & 15.49 & 0.0038 & August & 0.13 & 0.21 & 1.14 \\
\hline & & & & & Early July & -0.29 & 0.21 & 0.75 \\
\hline & & & & & June & 0.81 & 0.23 & 2.24 \\
\hline & & & & & Late July & -0.64 & 0.26 & 0.53 \\
\hline & & & & & Mid-July & 0.00 & 0.00 & 1.00 \\
\hline Previous crop & 266.30 & 3 & 181.28 & $<.0001$ & Barley & -3.07 & 0.34 & 0.05 \\
\hline & & & & & Legume & -1.55 & 0.20 & 0.21 \\
\hline & & & & & Tef & -0.82 & 0.23 & 0.44 \\
\hline & & & & & Wheat & 0.00 & 0.00 & 1.00 \\
\hline Weed & 234.24 & 2 & 32.06 & $<.0001$ & High (> 61 weeds $\mathrm{m}^{2}$ ) & 0.50 & 0.17 & 1.65 \\
\hline population & & & & & Low $\left(<40\right.$ weeds $\left.\mathrm{m}^{-2}\right)$ & 0.91 & 0.18 & 2.48 \\
\hline & & & & & $\begin{array}{l}\text { Medium ( } 41-60 \text { weeds } \\
\mathrm{m}^{-2} \text { ) }\end{array}$ & 0.00 & 0.00 & 1.00 \\
\hline Soil type & 222.7967 & 2 & 11.45 & 0.0033 & Red & 0.30 & 0.24 & 1.34 \\
\hline & & & & & Gray & 0.52 & 0.17 & 1.69 \\
\hline & & & & & Black & 0.00 & 0.00 & 1.00 \\
\hline
\end{tabular}

$d f$ degrees of freedom, $\operatorname{Pr}$ probability of a $X^{2}$ value exceeding the deviance reduction, $S E$ standard error 
were associated with the probability of lower disease incidence than that of Tikur jiru (Table 4).

Durum wheat severity was analyzed with reference to deviance, natural logarithms of odds ratio, and standard error of added variables in a reduced model (Table 4). Varieties were also tested in a reduced multiple model. High STB severity (> 25\%) had a high probability of association to Firno, Enzosh, and Nech sindie with 13.14, 13.1, and 3.95 times, respectively, greater probabilities that STB severity occurred than Tikur jiru.

\section{Association of septoria tritici blotch with cultural practices}

Cultural practices varied in their association to septoria tritici blotch incidence and severity. Cultural practices, such as previous crop, planting date, plant population, and weed density, were highly associated with incidence and severity when first entered as single variables into the model (Table 4).

The parameter estimates resulting from the reduced regression model and their standard errors were depicted (Table 4). There were about 1.56, 2.16, and 1.79 times higher probabilities that STB incidence exceeded 50\% in high plant population compared to medium plant population, June planting date compared to mid-July planting date, and high weed density fields compared to medium weed density fields, respectively. Low STB incidence ( $\leq$ $50 \%)$ and severity $(\leq 25 \%)$ had a higher probability of association to legume, barley, and tef previous crop than wheat. Similarly, high STB severity $(>25 \%)$ had a high probability of association to high plant population, June planting, and high weed population.

\section{Association of septoria tritici blotch with soil type}

In this survey, highly significant association of STB with soil type was observed. Septoria tritici blotch incidence and severity were positively associated with red soils compared to black soil type. The parameter estimates resulting from the reduced regression model indicated that high STB incidence $(>50 \%)$ had a high probability of association to red and gray soils than black soils (Table 4). There were about 2.55 and 2.21 times greater probabilities that STB incidence will exceed $50 \%$ in the red and gray soil types compared to black soil types, respectively.

\section{Discussion}

In this study, the prevalence and intensity of septoria tritici blotch varied among agronomic and environmental factors. This might be attributed to higher level of humidity at higher altitude (Janet and Tammy 2008) and highest level of infection on rainy cloudy with high relative humidity (Magboul et al. 1992; Kema et al. 1996; Dancer et al. 1999). Brown and Caligari (2008) stated that environmental variables of a macro nature have to be considered (Schulthesis et al. 1997), as well as other possible differences in the environment micro ones that affect individual plants, which leads to disease variation in district and altitude range.

A wide disease resistance variation occurred in different wheat genotypes for septoria tritici blotch (Gough 1978) and common bean genotypes for CBB (Fininsa and Yuen 2001a). In this regard, durum wheat genotypes had different disease resistance capacities with different agronomic features. This variation is important for breeding program to develop high yielder and STB resistant varieties (Gough 1978). In addition to genotype variability (Nadeem et al. 2008; Kahrizi et al. 2010), planting time also affects the development of septoria tritici blotch. The result of this study is in agreement with Shaw and Royle (1993) that crops sown at early times pose greater risks of infection. This may be due to the fact that early sown plants produce more leaves and result in greater presence of inoculums (Shaw and Royle 1993). In addition, slower stem extension in early sown crops gives more time for the infection to move from older to younger leaves (Shaw and Royle 1993; Lovell et al. 2004).

The result of this study is in accordance with Agrios (2005); some plants escape disease because they are susceptible to a pathogen only at a particular growth stage (young leaves, stems, or fruits; at blossoming or fruiting; at maturity and early senescence); therefore, if the pathogen is absent or inactive at that particular time, such plants avoid becoming infected, but latent infection may occur.

The result of present survey showed that high plant population, high weed density, wheat as a previous crop, and early planting favor the development of STB disease. A number of studies reported that crop rotation is important in reducing the incidence and severity of diseases. Previously, Krupinsky (1999) recommended crop rotation as a means to increase decomposition of the infected crop residue while non-host crops are grown. The author stated that crop rotation might not eliminate the disease; however, they would reduce the inoculum level of the pathogen. In addition, Pedersen and Hughes (1992) reported that crop rotation of at least 2 years between wheat crops was required to achieve adequate control of the septoria disease complex under ideal environmental conditions, while a 1-year break between wheat crops was effective in reducing disease severity under conditions that were unfavorable for the pathogen. The reduction of the disease is attributed to the contribution of wheat or related crops to the build of STB pathogen, while rotation with a non-susceptible crop reduces the pathogen inoculum levels as it helps to break the cycle. The disease severity of leaf spot pathogens is higher in wheat grown after wheat than after flax 
or lentil although the effects are only obvious in years with high disease pressure (Fernandez et al. 1998).

It demonstrated the effect of planting date on the diseases that corresponded with previous reports (Shaner et al. 1975; Thomas et al. 1989; Shaw and Royle 1993) that crops sown at early times pose greater risks of infection. A higher disease intensity in early sown fields might be attributed to much amount of leaves in early sown plants (Shaw and Royle 1993; Tesfaye 1988; Zelalem 1989), which, in turn, results in greater presence of inocula (Shaner et al. 1975; Shaw and Royle 1993), and slower stem extension gives more time for the infection to move from older to younger leaves (Shaw and Royle 1993; Lovell et al. 2004). In addition, too longer maturation time gives rise to another STB cycle of multiplication in diseased plants (Shaw and Royle 1993).

In accordance to the result of the present study, previous reports showed that high plant population favors the development of plant pathogen. Fininsa and Yuen (2001a) reported low maize rust incidence had a high probability of association to low maize crop density. This is attributed to high seeding rates, and narrow row spacing creates a favorable microclimate for the development of stagonospora nodorum blotch and septoria tritici blotch (Tompkins et al. 1993). On the contrary, reduced plant density in the field increased nutrient availability, which, in turn, aggravating the disease (Rodgers-Gray and Shaw 2000), and increased the dispersal ability of STB spores by rain-splash (Shaw and Royle 1993).

The influence of weed density on the intensity of the disease was observed in this study. The result of this study is supported by the previous work (Woldeab et al. 2007) which reported that barley leaf rust (Puccinia hordei) incidence increases with an increase in weed population. In this connection, Duczek et al. (1996) discussed that dense population of weeds in a field may contribute to disease development by increasing the density of the plant canopy or serving as an inoculum reservoir in the absence of a susceptible host crop.

Similarly, STB severity was significantly associated with soil type (Table 5). Walters and Bingham (2007) and Ruska et al. (1997) discussed that soil factors, such as organic matter, red content, nutrients, and soil $\mathrm{pH}$, have an impact on yield, plant diseases, and interactions. Similarly, Saito et al. (2006) reported the availability of moisture in heavy soils (red soils) due to high water holding capacity favors the development of the disease.

\section{Conclusions}

Our results show environmental variables, cultural practice, and responses were important for the development of STB. These findings indicate that proper plant density, weed management, and plant growth stage are crucial to reduce the impact of septoria tritici blotch in wheat. Disease intensity of septoria tritici blotch varied among districts, altitude, varieties, growth stage of the crop, cropping practices, and soil type. High STB incidence and severity were positively associated with varieties, high plant population, high weed density, early (June) planting, late milk stage, and red soil type, whereas previous crop was positively associated with low disease incidence and severity. As a result, different epidemiologically relevant factors must be considered to manage STB disease. Therefore, these factors must be considered in designing strategies for the effective management of septoria tritici blotch.

\section{Abbreviations \\ DR: Deviance reduction; LRT: Likelihood ratio test; masl: Meter above sea level; Pr: Probability; STB: Septoria tritici blotch; Subsp.: Subspecies; $x^{2}$ : Chi- square}

\section{Acknowledgements}

We appreciate Amhara Agricultural Research Institute and Sirinka Agricultural Research Center for providing materials for this study.

\section{Authors' contributions}

$\mathrm{BN}, \mathrm{YG}$, and $\mathrm{AA}$ designed the experiment. BN conducted the data collection, analyzed the data, and wrote the manuscript. YG and AA revised the manuscript. All authors read and approved the final manuscript.

\section{Funding}

There was no funding for this project.

\section{Availability of data and materials Not applicable}

Ethics approval and consent to participate

Not applicable

\section{Consent for publication}

Not applicable

\section{Competing interests}

The authors declare that they have no competing interests.

\section{Author details}

${ }^{1}$ Sirinka Agricultural Research Center, Woldia, Ethiopia. ${ }^{2}$ Bioversity International, Addis Ababa, Ethiopia. ${ }^{3}$ Partnership for Aflatoxin Control in Africa, Addis Ababa, Ethiopia.

Received: 28 November 2019 Accepted: 8 September 2020 Published online: 09 November 2020

\section{References}

Agrios GN (2005) Plant pathology, 5th edn. Acadamic Press, New York, p 922

Blixt S (1988) Genetic resources at work: practical examples of the importance of gene bank work regarding agriculture. Horticulture and Forestry Brief report. Hereditas 108:271-273

Brown J, Caligari PDS (2008) An introduction to plant breeding. Blackwell Publishing, U.K., p 209

Cherdouh AD, Khelifi JM, Carrillo, Nieto-Taladriz MT (2005) The high and low molecular weight glutenin subunit polymorphism of Algerian durum wheat landraces and old cultivars. Plant Breed 124:338-342

CSA (Central Statistical Agency) (2009) Agricultural Sample Survey 2009/2010. Reports on forecast of area and production of major crops: rural private peasant holding 'Meher' season. Statistical Bulletin, Addis Ababa, p 439

Damania AB, Hakim S, Moualla MY (1992) Evaluation of variation in Triticum dicoccum for wheat improvement in stress environments. Hereditas 116:163166 
Dancer J, Daniels A, Cooley N, Foster S (1999) Septoria tritici and Stagonospora nodorum as model pathogens for fungicide discovery. In: Lucas JA, Bowye $P$, Anderson HM (eds) Septoria on cereals: a study of pathosystems. CAIB Publishing, UK

Devra IJ, Hodgkin T (1999) Wild relatives and crop cultivars: detecting natural introgression and farmer selection of new genetic combinations in agroecosystems. Mol Ecol 8:159-173

Duczek LJ, Jones-Flory LL, Reed SL, Bailey KL, Lafond GP (1996) Sporulation of Bipolaris sorokiniana on the crowns of crop plant grown in Saskatchewan. Can J Plant Sci 76:861-867

Eyal Z, Scharen AL, Huffman MD, Prescott LM (1985) Global insights into virulence frequencies of Mycosphaerella graminicola. Phytopathology 75 : $1456-1462$

Eyal Z, Scharen AL, Prescott JM, van Ginkel M (1987) The septoria diseases of wheat: concepts and methods of disease management. CIMMYT, Mexico

Fernandez MR, Zentner RP, McConkey BG, Campbell CA (1998) Effects of crop rotations and fertilizer management on leaf spotting diseases of spring wheat in southwestern Saskatchewan. Can J Plant Sci 78:489-496

Fininsa C, Yuen J (2001a) Association of bean rust and common bacterial blight epidemics with cropping systems in Hararghe highlands, eastern Ethiopia. Int J Pest Manag 47:211-219

Fininsa C, Yuen J (2001b) Association of maize rust and leaf blight epidemics with cropping systems in Hararghe highlands, eastern Ethiopia. Crop Prot 20: 669-678

Ganeva G, Korzun V, Landjeva S, Popova Z, Christov NK (2011) Genetic diversity assessment of Bulgarian durum wheat (Triticum durum Desf.) landraces and modern cultivars using microsatellite markers. Genet Resour Crop Evol 57: 273-285

Gough FG (1978) Effect of wheat host cultivars on pycnidiospore production by Septoria tritici. Phytopathology 68:1343-1345

Grausgrubera H, Oberforsterb M, Ghambashidzec G, Ruckenbauera P (2005) Yield and agronomic traits of Khorasan wheat (Triticum turanicum Jakubz.). Field Crop Res 91:319-327

Janet B, Tammy H (2008) Garlic: organic production. www.attra.ncat.org/attra-pub. Accessed on 18/01/2013.

Kahrizi D, Cheghamriza K, Kakaei M, Mohammadi R, Ebadi A (2010) Heritability and genetic gain of some morpho-physiological variables of durum wheat (Triticum turgidum var. durum). Afr J Biotechnol 9(30):4687-4691

Kema GHJ, Yu D, Rijkenberg FHJ, Shaw MW, Baayen RP (1996) Histology of the pathogenesis of Mycosphaerella graminicola in wheat. Phytopathology 86(7): 777-785

Krupinsky JM (1999) Influence of cultural practices on Septoria/Stagonospora diseases. In: van Ginkel M, McNab A, Krupinsky L (eds) Septoria and Stagonospora diseases of cereals: a compilation of global research. CIMMYT, Mexico, pp 105-110

Lovell DJ, Hunter T, Powers SJ, Parker SR, Van den Bosch F (2004) Effect of temperature on latent period of septoria leaf blotch on winter wheat under outdoor conditions. Plant Pathol:170-181

Magboul AM, Geng S, Gilchrist DG, Jackson LF (1992) Environmental influence on the infection of wheat by Mycosphaerella graminicola. Phytopathology 82 1407-1413

McCullagh P, Nelder JA (1989) Generalized linear models, second edn. Chapman and Hall, London, p 511

Nadeem SA, Haque MI, Ahmedani MS, Rauf A, Munir M, Firdous SS, Rattu A-U-R, Ahmad I (2008) Impact of stripe rust on kernel weight of wheat varieties sown in rainfed areas of Pakistan. Pak J Bot 40(2):923-929

Pedersen EA, Hughes GR (1992) The effect of crop rotation on development of the septoria disease complex on spring wheat in Saskatchewan. Can J Plant Pathol 14:152-158

Polley RW, Thomas MR (1991) Surveys of diseases of winter wheat in England and Wales, 1976-1988. Ann Appl Biol 119:1-20

Ponomarenko A, Goodwin SB, Kema GHJ (2011) Septoria tritici blotch (STB) of wheat. Plant Health Instructor. https://doi.org/10.1094/PHI-I-2011-0407-01

Rajaram S, Dubin HJ (1977) Avoiding genetic vulnerability in semi-dwarf wheats. Ann N Y Acad Sci 287:243-254

Rodgers-Gray BS, Shaw MW (2000) Substantial reductions in winter wheat diseases caused by addition of straw but not manure to soil. Plant Pathol 49: 590-599

Ruska PK, Buruchara RA, Gatabazi, Pastor-Corrales MA (1997) Occurance and distribution in Rwanda of soil borne fungi pathoginic to the common bean. Plant Dis 81:445-449
Saari EE, Prescott JM (1975) A scale for appraising the foliar intensity of wheat disease. Plant Dis Rep 59:377-380

Saari EE, Wilcoxson RD (1974) Plant disease situation of high-yielding dwarf wheats in Asia and Africa. Annu Rev Phytopathol 12:49-68

Saito K, Linquist B, Keobualapha B, Shiraiwa T, Horie T (2006) Farmers' knowledge of soils in relation to cropping practices: a case study of farmers in upland rice based slash-and-burn systems of northern Laos. Geoderma 136:64-74

SAS Institute (1993) SAS Technical Report P/243, SAS/STAT soft/ware, The GENMOD Procedure Release 6.09. SAS Institute Inc, Cary, p 88

SAS Institute (2003) SAS User's Guide: Statistics. Version 9.1. SAS Institute Inc, Cary, $\mathrm{NC}$

Schulthesis U, Feil B, Jutzi SC (1997) Yield-independent variation in grain nitrogen and phosphorus concentration among Ethiopian wheats. Agron J 89(3):497-506

Shaner G, Finney RE, Patterson FL (1975) Expression and effectiveness of resistance in wheat to Septoria tritici blotch. Phytopathology 65:761-766

Sharma RC, Duveiller E (2007) Advancement toward new spot blotch resistant wheats in South Asia. Crop Sci 47:961-968

Shaw MW, Royle DJ (1993) Factors determining the severity of epidemics of Mycosphaerella graminicola (Septoria tritici) on winter wheat in the UK. Plant Pathol 42:882-899

Tesfaye T (1988) Durum wheat breeding in Ethiopia. In: Ginkel MV, Tanner DG (eds) The Fifth Regional Wheat Workshop for Eastern, Central and Southern Africa and the Indian Ocean, Antisirabe, Madagascar, October 5-10, p 1987

Thomas MR, Cook RJ, King JE (1989) Factors affecting development of Septoria tritici in winter wheat and its effect on yield. Plant Pathol 38:246-257

Tompkins DK, Fowler DB, Wright AT (1993) Influence of agronomic practices on canopy microclimate and septoria development in no-till winter wheat produced in the parkland region of Saskatchewan. Can J Plant Sci 73:331-344

Walters DR, Bingham IJ (2007) Influence of nutrition on disease development caused by fungal pathogens: implications for plant disease control. Ann Appl Biol 151:307-324

Woldeab G, Yuen J, Fininsa C, Singh H (2007) Barley leaf rust (Puccinia hordei Otth) in three production systems and practices in Ethiopia. Crop Prot 26: 1193-1202

Yahyaoui N, Brunner S, Keller B (2006) Rapid generation of new powdery mildew resistance genes after wheat domestication. Plant J 47:85-89

Zadoks JC, Schein RD (1979) Epidemiology and plant disease management. Oxford University Press, New York, p 427

Zelalem A (1989) Relationship between spike morphology, hordeins and altitude within Ethiopian barley, Hordeum vulgare L. (Poaceae). Hereditas 110:203-209

Zewde T, Fininsa C, Sakhuja PK, Ahmed S (2007) Association of white rot ( Sclerotium cepivorum) of garlic with environmental factors and cultural practices in the North Shewa highlands of Ethiopia. Crop Prot 26:1566-1573

\section{Publisher's Note}

Springer Nature remains neutral with regard to jurisdictional claims in published maps and institutional affiliations.

\section{Submit your manuscript to a SpringerOpen ${ }^{\circ}$ journal and benefit from:}

- Convenient online submission

- Rigorous peer review

- Open access: articles freely available online

- High visibility within the field

- Retaining the copyright to your article

Submit your next manuscript at $>$ springeropen.com 